\title{
PROMs suggest a festival of benefits in LATITUDE
}

Analysis of patient-reported outcome measures (PROMs) in the LATITUDE trial shows a substantial improvement in health-related quality of life (HRQOL) in men with metastatic prostate cancer with the addition of abiraterone and prednisone to androgen deprivation therapy (ADT).

The multicentre, randomized, phase III trial included men with newly diagnosed, high-risk, metastatic castration-naive prostate cancer. Patients were randomized to receive daily ADT plus $1000 \mathrm{mg}$ oral abiraterone and $5 \mathrm{mg}$ oral prednisone or ADT plus placebo. Overall survival and radiographic-progression-free survival were the coprimary end points of LATITUDE and have been previously reported, showing significant improvements with the addition of abiraterone and prednisone. The LATITUDE investigators also collected PROMs data regarding pain, fatigue, symptoms, and HRQOL using the Brief Pain Inventory-Short Form (BPI-SF), Brief Fatigue Inventory, Functional Assessment of Cancer Therapy Prostate scale, and EuroQol five-dimensions, five-levels questionnaire throughout the study.

The BPI-SF questionnaire data showed that patients receiving ADT plus abiraterone and prednisone had a longer median time to worst pain intensity progression than those who received ADT plus placebo. Median time to pain interference progression was not reached in the combination group compared with 18.4 months $(P<0.0001)$ in the ADT alone group. Improvements were seen in changes from baseline in worst pain intensity, pain interference, and average pain progression with ADT plus abiraterone and prednisone compared with ADT plus placebo at most time points. Changes from baseline in worst

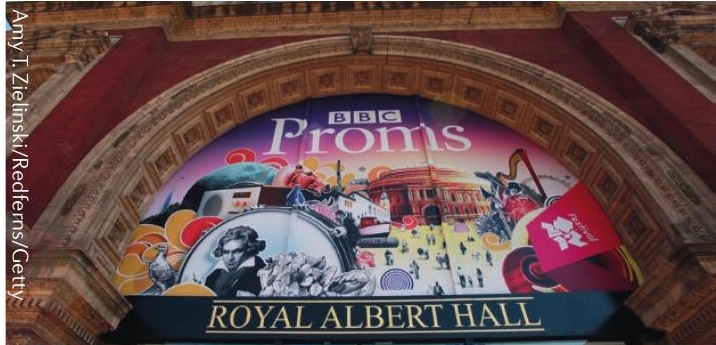

fatigue scores were also improved in men who received combination therapy compared with ADT plus placebo.

These LATITUDE PROMs data are in accordance with survival data previously reported, as well as data reported in the STAMPEDE trial. The addition of abiraterone and prednisone to ADT not only improves survival but also has significant benefits on HRQOL, suggesting that this treatment regimen could be considered a new standard of care for metastatic prostate cancer.

\section{Annette Fenner}

ORIGINAL ARTICLE Chi, K. N. et al. Patient-reported outcomes following abiraterone acetate plus prednisone added to androgen deprivation therapy in patients with newly diagnosed metastatic castration-naive prostate cancer (LATITUDE): an international, randomised phase 3 trial. Lancet Oncol. https://doi.org/10.1016/S1470-2045(17)30911-7 (2018) 